\title{
Synthesis of a CGRP Receptor Antagonist via an Asymmetric Synthesis of 3-Fluoro-4- aminopiperidine
}

Carmela Molinaro, ${ }^{*}$ Eric M. Phillips, ** Bangping Xiang, *t Erika Milczek, * Michael Shevlin, * Jaume Balsells, + Scott Ceglia, ${ }^{*}$ Jiahui Chen, ${ }^{ \pm}$Lu Chen, ${ }^{ \pm}$Qinghao Chen, ${ }^{*}$ Zhongbo Fei, $*$ Scott Hoerrner, ${ }^{*} \mathrm{Ji}$ Qi, ${ }^{ \pm}$Manuel de Lera Ruiz, ${ }^{\star}$ Lushi Tan,${ }^{*}$ Baoqiang Wan,${ }^{ \pm}$Jingjun Yin, ${ }^{*}$

${ }^{\ddagger}$ Process Research \& Development, Merck \& Co., Inc., 126 E. Lincoln Avenue, Rahway, New Jersey 07065, United States

†Process Research \& Development, Merck \& Co., Inc., 770 Sumneytown Pike, West Point, PA 19486, United States

${ }^{ \pm}$STA Pharmaceutical R\&D Co., Ltd, 90 Delin Road, Waigaoqiao Free Trade Zone, Shanghai 200131, China

eric.phillips@merck.com,bangping_xiang@merck.com 


\section{Supporting Information}

\section{Contents}

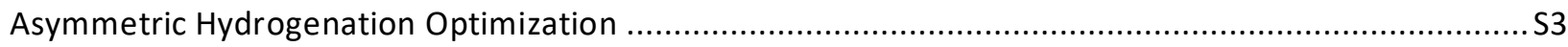

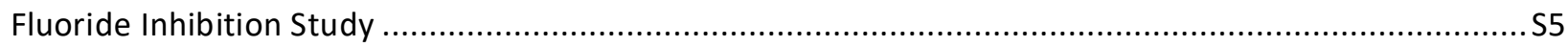

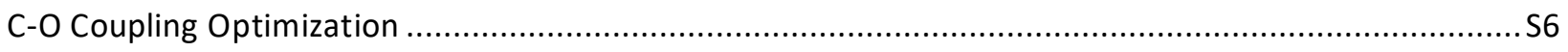

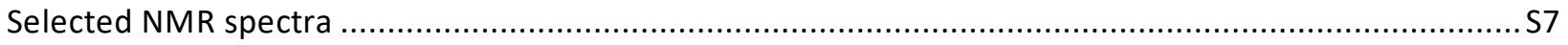

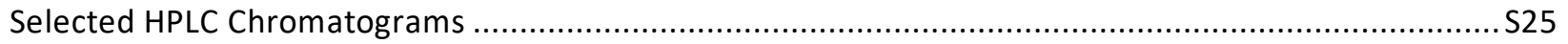




\section{Asymmetric Hydrogenation Optimization}

\section{General Microscale Asymmetric Hydrogenation Procedure}

Microscale reactions were carried out in $8 \times 30 \mathrm{~mm}$ glass vial inserts in aluminum 96-well microtiter plates. Solutions of metal precursor and ligand were stirred at r.t. in DCE for 15 min. $\mathrm{HBF}_{4} \cdot \mathrm{OEt}_{2}$ was added as a DCE solution to ruthenium catalysts and the mixtures were stirred for an additional $15 \mathrm{~min}$ at r.t. followed by removal of the volatiles on a vacuum centrifuge. Solutions of the substrate were introduced, and the plates were sealed in pressure vessels and removed from the glovebox. The pressure vessels were attached to a gas manifold and the supply line and vessel headspace were purged several times with nitrogen, followed by three purges with hydrogen. The vessel was pressurized to the desired pressure with hydrogen, and heated to the reaction temperature with $500 \mathrm{rpm}$ shaking. After the desired reaction time, the vessel was vented and the reactions were sampled for analysis.

\section{Ligand Screening Experiments}

The general microscale asymmetric hydrogenation procedure was followed using $0.4 \mu \mathrm{mol}$ of $(\mathrm{NBD})_{2} \mathrm{RhBF}_{4},(\mathrm{COD}) \mathrm{Ru}(2-\text { methylallyl })_{2}\left(+0.8 \mu \mathrm{mol} \mathrm{HBF}_{4} \cdot \mathrm{OEt}_{2}\right)$, or $(\mathrm{COD})_{2} \mathrm{IrBF}_{4}, 0.42 \mu \mathrm{mol}$ of 192 chiral phosphine ligands, $1.6 \mu \mathrm{mol}$ substrate, $1.6 \mu \mathrm{mol} \mathrm{HBF}_{4} \cdot \mathrm{OEt}_{2}, 100 \mu \mathrm{L} \mathrm{MeOH}, 500$ psi $\mathrm{H}_{2}, 40{ }^{\circ} \mathrm{C}, 18 \mathrm{~h}$. 
TABLE S1: Top Screening Results and Optimization of Asymmetric Hydrogenation of 8.

\begin{tabular}{|c|c|c|c|c|c|c|c|c|c|}
\hline Entry & $\mathrm{M}$ & $\begin{array}{c}\mathrm{M} \\
\mathrm{mol} \%)\end{array}$ & Ligand & Solvent & Conc & 8 & $\begin{array}{l}\text { Ratio } \\
: 12 \text { : }\end{array}$ & 14 & $\begin{array}{c}12 \\
(\% \text { ee })\end{array}$ \\
\hline $1^{a}$ & Ir & 25 & A121-1 & $\mathrm{MeOH}$ & 0.016 & 18 & 7 & 75 & 99 \\
\hline $2^{a}$ & Ir & 25 & N013-1 & $\mathrm{MeOH}$ & 0.016 & 0 & 12 & 88 & -99 \\
\hline $3^{a}$ & Ir & 25 & J502-1 & $\mathrm{MeOH}$ & 0.016 & 35 & 15 & 50 & -97 \\
\hline $4^{a}$ & Ir & 25 & J506-1 & $\mathrm{MeOH}$ & 0.016 & 62 & 6 & 32 & -96 \\
\hline $5^{a}$ & $\mathrm{Rh}$ & 25 & T027-2 & $\mathrm{MeOH}$ & 0.016 & 0 & 70 & 30 & 97 \\
\hline $6^{a}$ & $\mathrm{Rh}$ & 25 & T021-2 & $\mathrm{MeOH}$ & 0.016 & 0 & 50 & 50 & 90 \\
\hline $7^{a}$ & $\mathrm{Rh}$ & 25 & T025-2 & $\mathrm{MeOH}$ & 0.016 & 0 & 46 & 54 & 89 \\
\hline $8^{a}$ & $\mathrm{Rh}$ & 25 & W023-1 & $\mathrm{MeOH}$ & 0.016 & 0 & 26 & 74 & 87 \\
\hline $9^{a}$ & $\mathrm{Ru}$ & 25 & M003-1 & $\mathrm{MeOH}$ & 0.016 & 0 & 93 & 7 & 86 \\
\hline $10^{a}$ & $\mathrm{Ru}$ & 25 & A108-1 & $\mathrm{MeOH}$ & 0.016 & 0 & 91 & 9 & 86 \\
\hline $11^{b}$ & $\mathrm{Ru}$ & 10 & A108-1 & $\mathrm{MeOH}$ & 0.02 & 61 & 36 & 3 & 60 \\
\hline $12^{b}$ & $\mathrm{Ru}$ & 10 & A108-1 & TFE & 0.02 & 7 & 87 & 6 & 12 \\
\hline $13^{b}$ & Ru & 10 & A108-1 & DCE & 0.02 & 0 & 95 & 5 & 43 \\
\hline $14^{b}$ & $\mathrm{Ru}$ & 10 & A108-1 & EtOAc & 0.02 & 1 & 82 & 17 & 83 \\
\hline $15^{b}$ & $\mathrm{Ru}$ & 10 & A108-1 & THF & 0.02 & 1 & 94 & 5 & 79 \\
\hline $16^{b}$ & $\mathrm{Ru}$ & 3 & A108-1 & THF & 0.2 & 1 & 96 & 3 & 86 \\
\hline $17^{b}$ & $\mathrm{Ru}$ & 3 & A108-1 & 2-Me-THF & 0.2 & 1 & 97 & 2 & 88 \\
\hline $18^{b}$ & $\mathrm{Ru}$ & 1 & A108-1 & 2-Me-THF & 0.2 & 26 & 72 & 2 & 85 \\
\hline $19^{b, d}$ & $\mathrm{Ru}$ & 1 & A108-1 & 2-Me-THF ${ }^{c}$ & 0.2 & 15 & 82 & 3 & 86 \\
\hline $20^{b, e}$ & $\mathrm{Ru}$ & 1 & A108-1 & 2-Me-THF ${ }^{c}$ & 0.2 & 0 & 97 & 3 & 86 \\
\hline
\end{tabular}

Conditions: a) $25 \mathrm{~mol} \%(\mathrm{COD})_{2} \mathrm{IrBF}_{4}$, (NBD) ${ }_{2} \mathrm{RhBF}_{4}$ or (COD)Ru(Meallyl $)_{2}+2 \mathrm{HBF}_{4} \cdot \mathrm{OEt}_{2} / 192$ chiral ligands, 1 equiv $\mathrm{HBF}_{4} \cdot \mathrm{OEt}_{2}, \mathrm{MeOH}$, $0.016 \mathrm{M}, 500 \mathrm{psi} \mathrm{H}_{2}, 40{ }^{\circ} \mathrm{C}$. b) (COD)Ru(Me-allyl) $)_{2} / \mathrm{A} 108-1$ / $\mathrm{HBF}_{4} \cdot \mathrm{OEt}_{2}(1: 1.05: 2)$, 1 equiv $\mathrm{HBF}_{4} \cdot \mathrm{OEt}_{2}, 500 \mathrm{psi}_{2}, 40{ }^{\circ} \mathrm{C}$. c) $10 \% \mathrm{v} / \mathrm{v} \mathrm{MeOH}$ added. d) 0.2 equiv $\mathrm{Ti}(\mathrm{OiPr})_{4}$ added. e) 2 equiv $\mathrm{Ti}(\mathrm{OiPr})_{4}$ added and 1.1 equiv $\mathrm{HBF}_{4} \cdot \mathrm{OEt}_{2}$ used. 


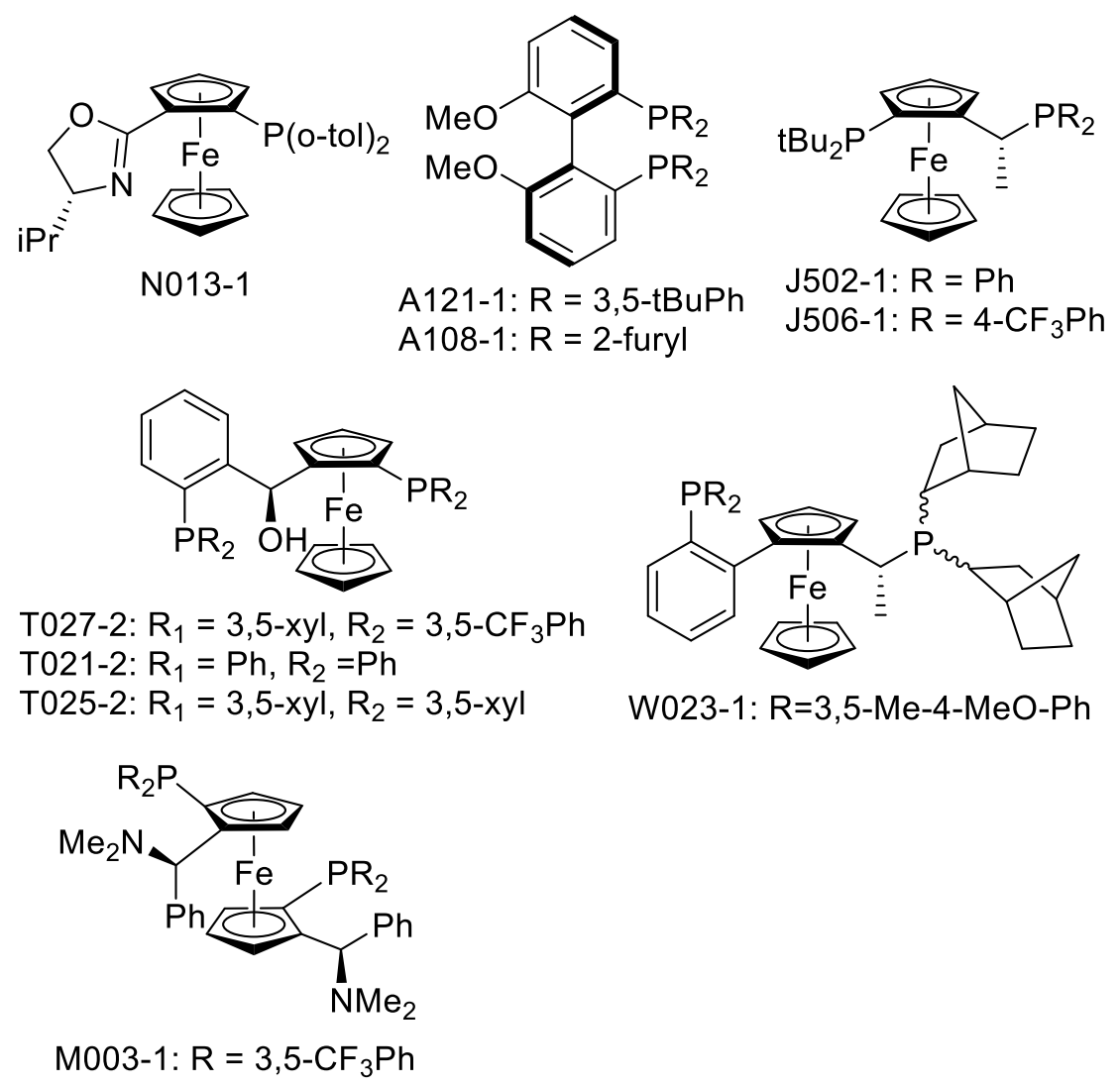

FIGURE S1: Structure of the Ligands used in the Asymmetric Hydrogenation of 24

\section{Fluoride Inhibition Study}

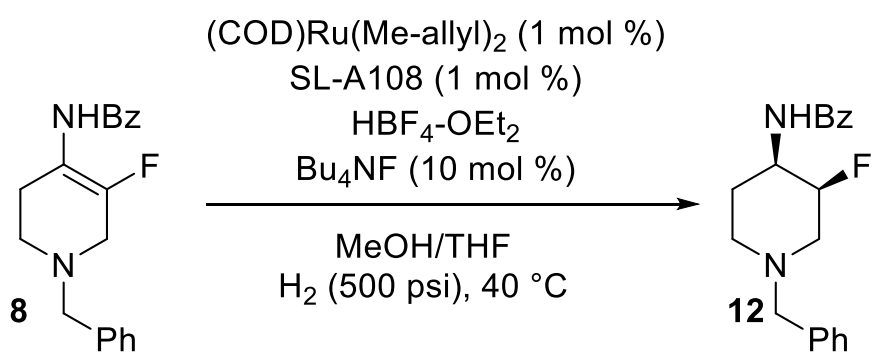

FIGURE S2: Structure of the Ligands used in the Asymmetric Hydrogenation

Following the general microscale asymmetric hydrogenation procedure, a controlled amount of Bu4NF was charged to each well. Reactions mixed for $18 \mathrm{~h}$. 
TABLE S2. Screening of Bu 4 NF charge

\begin{tabular}{ccc}
\hline Entry & $\mathbf{B u}_{4} \mathbf{N F}$ charge & Conversion (\%) \\
\hline $\mathbf{1}$ & $2 \mathrm{~mol} \%$ & 86.9 \\
$\mathbf{2}$ & $5 \mathrm{~mol} \%$ & 79.7 \\
$\mathbf{3}$ & $10 \mathrm{~mol} \%$ & 65.4 \\
\hline
\end{tabular}

\section{C-O Coupling Optimization}

\section{TABLE S3. Cu-Catalyzed C-O Coupling}

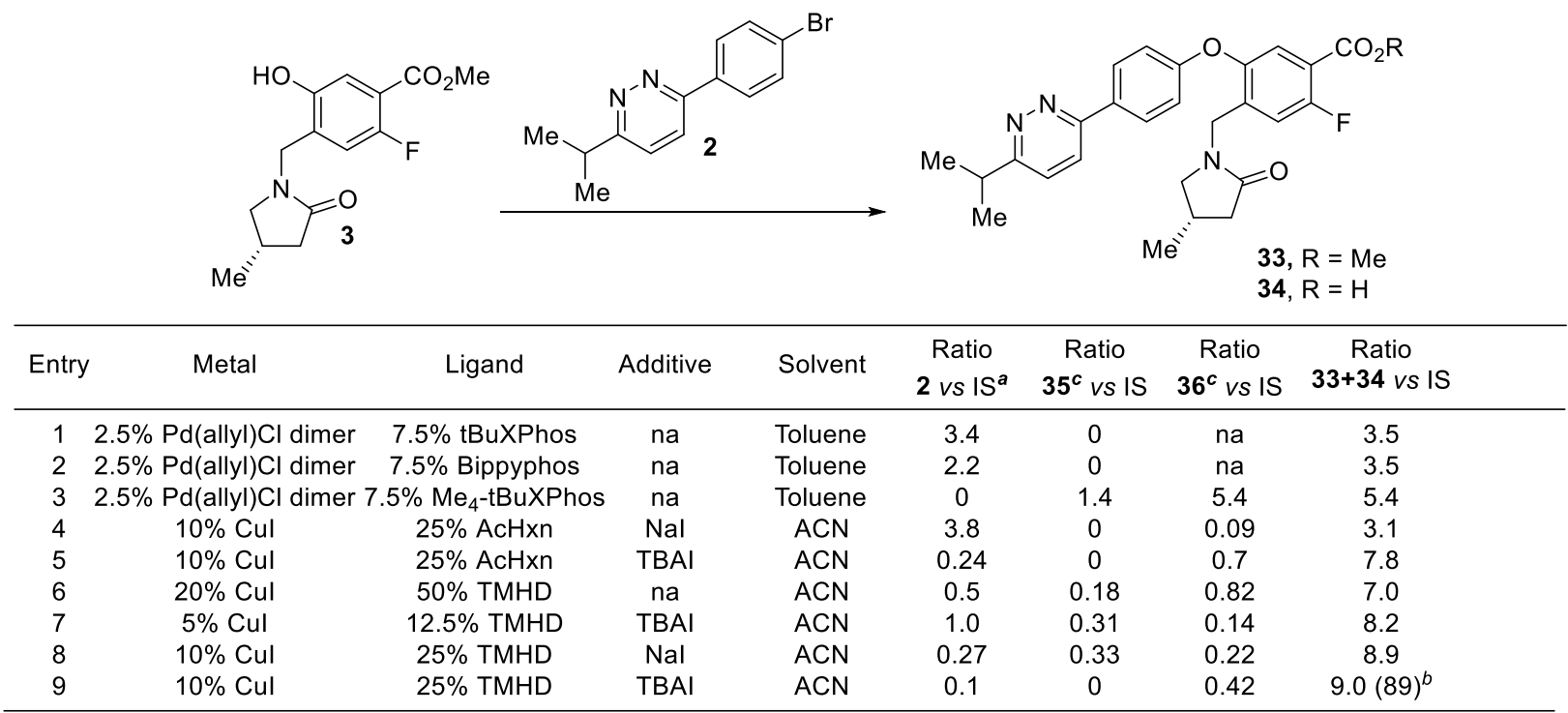

Conditions : a) Diphenyl was used as an Internal Standard b) AY determined by reverse phase HPLC

c) Structures of by-products

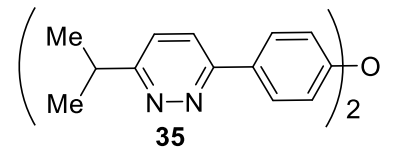<smiles>CC(C)c1ccc(-c2ccccc2)nn1</smiles> 


\section{Selected NMR spectra}
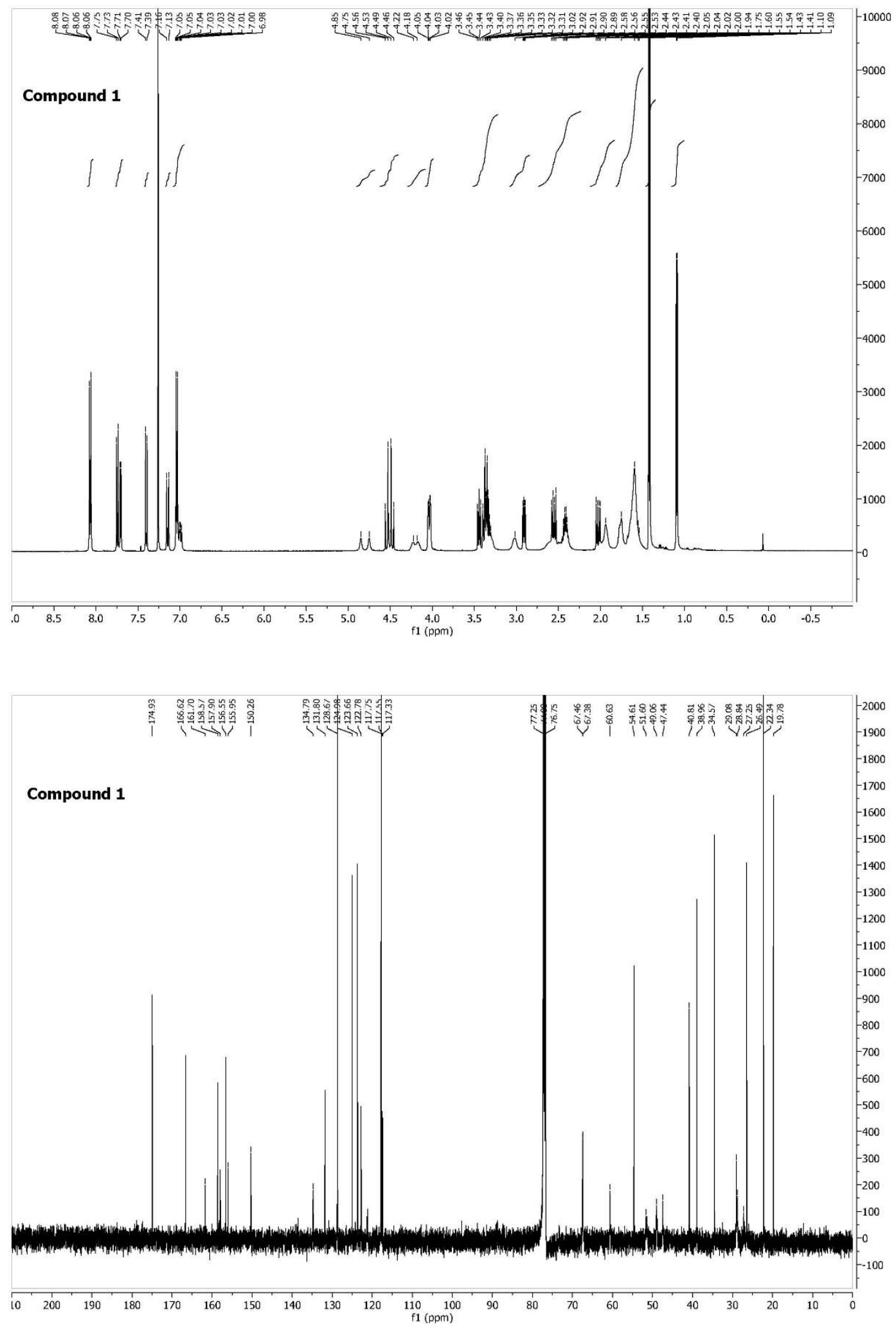

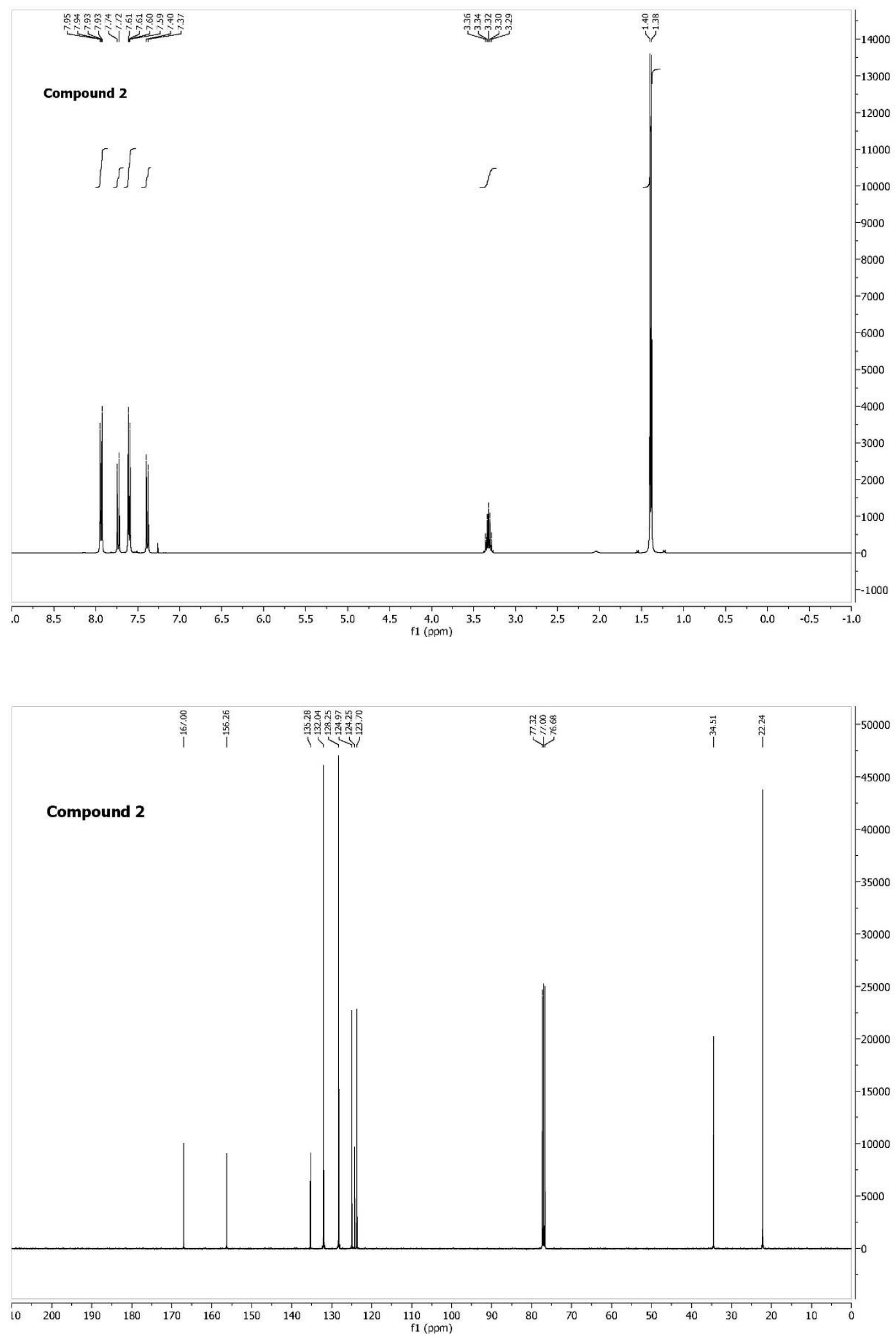

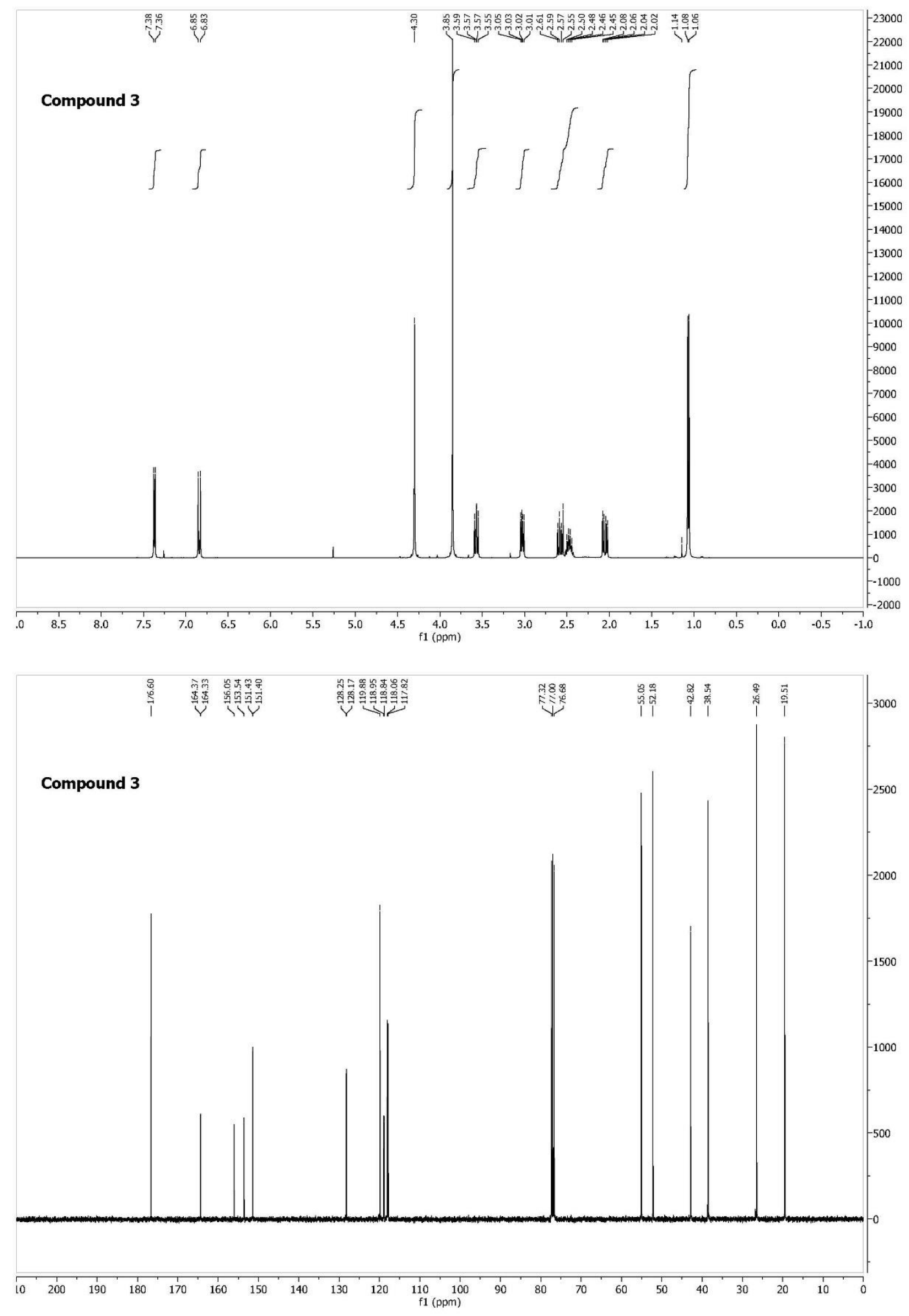

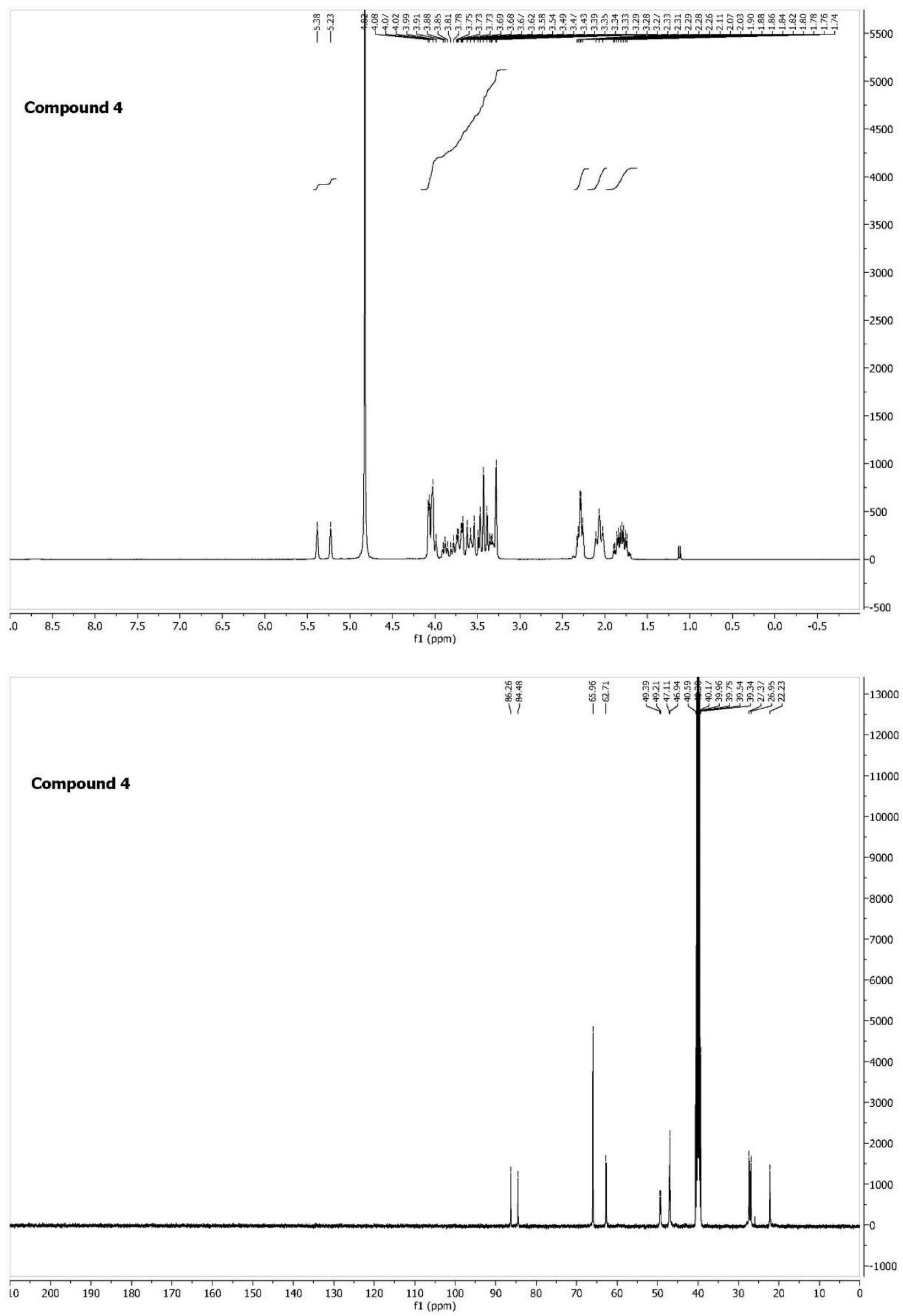

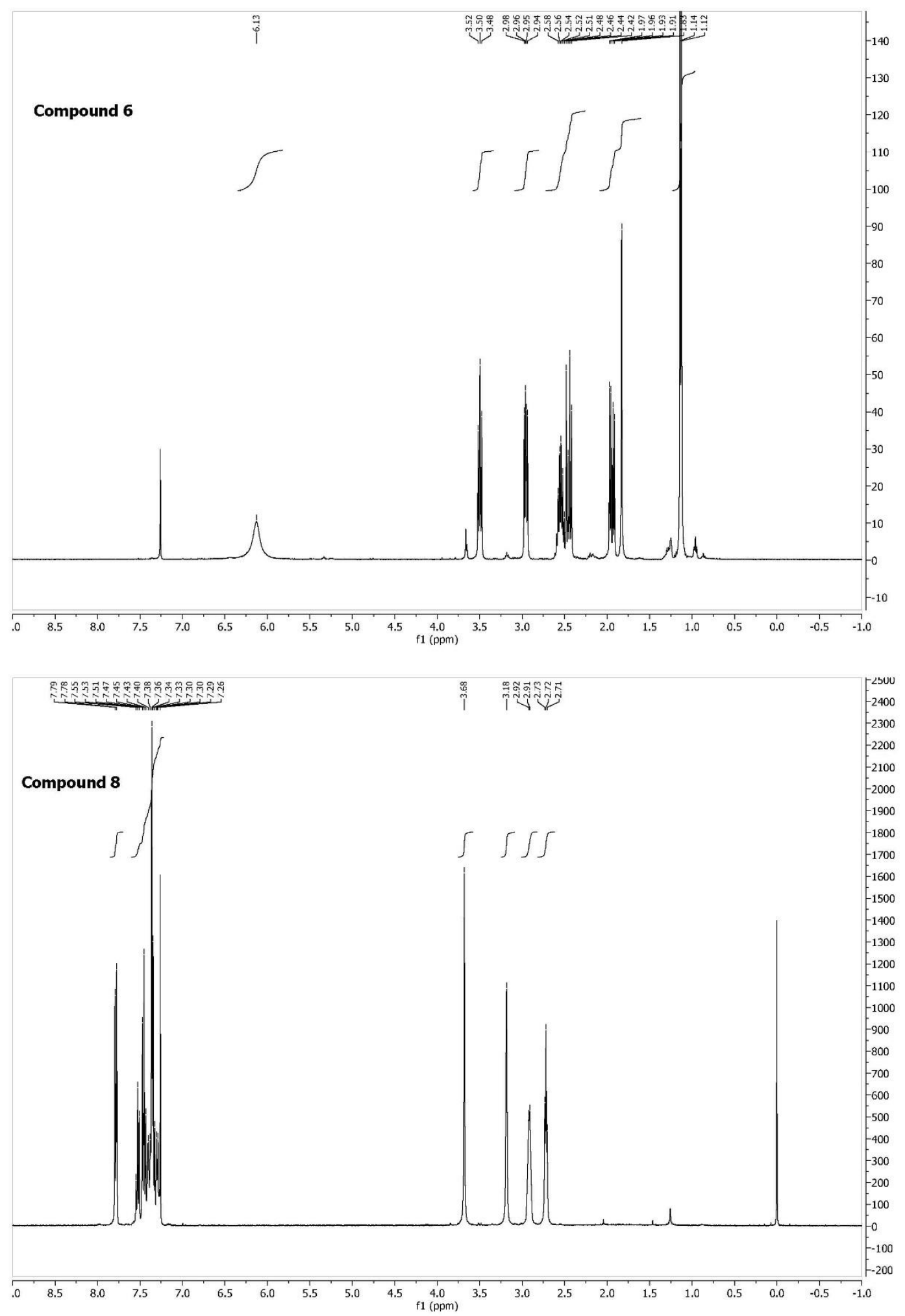


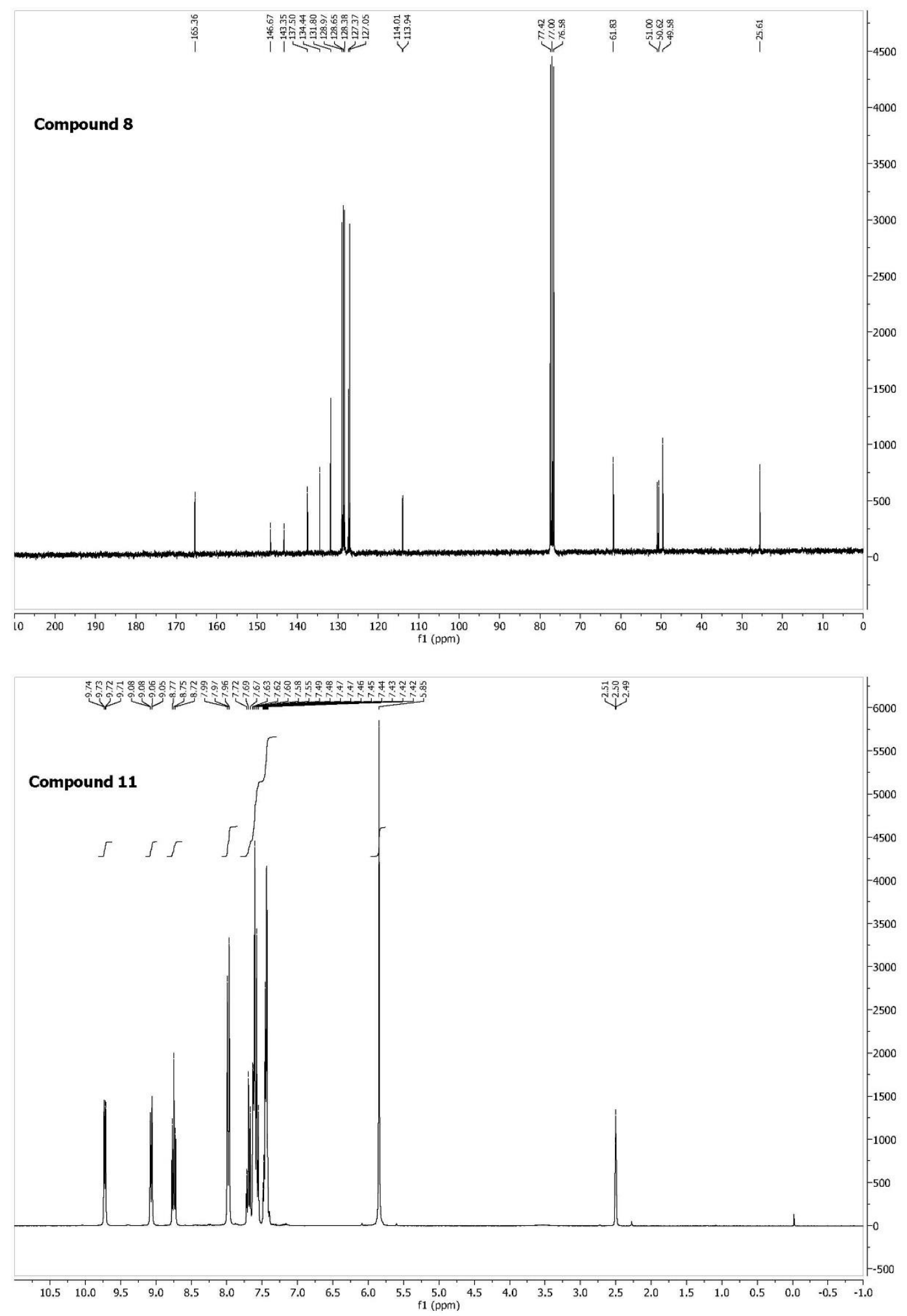




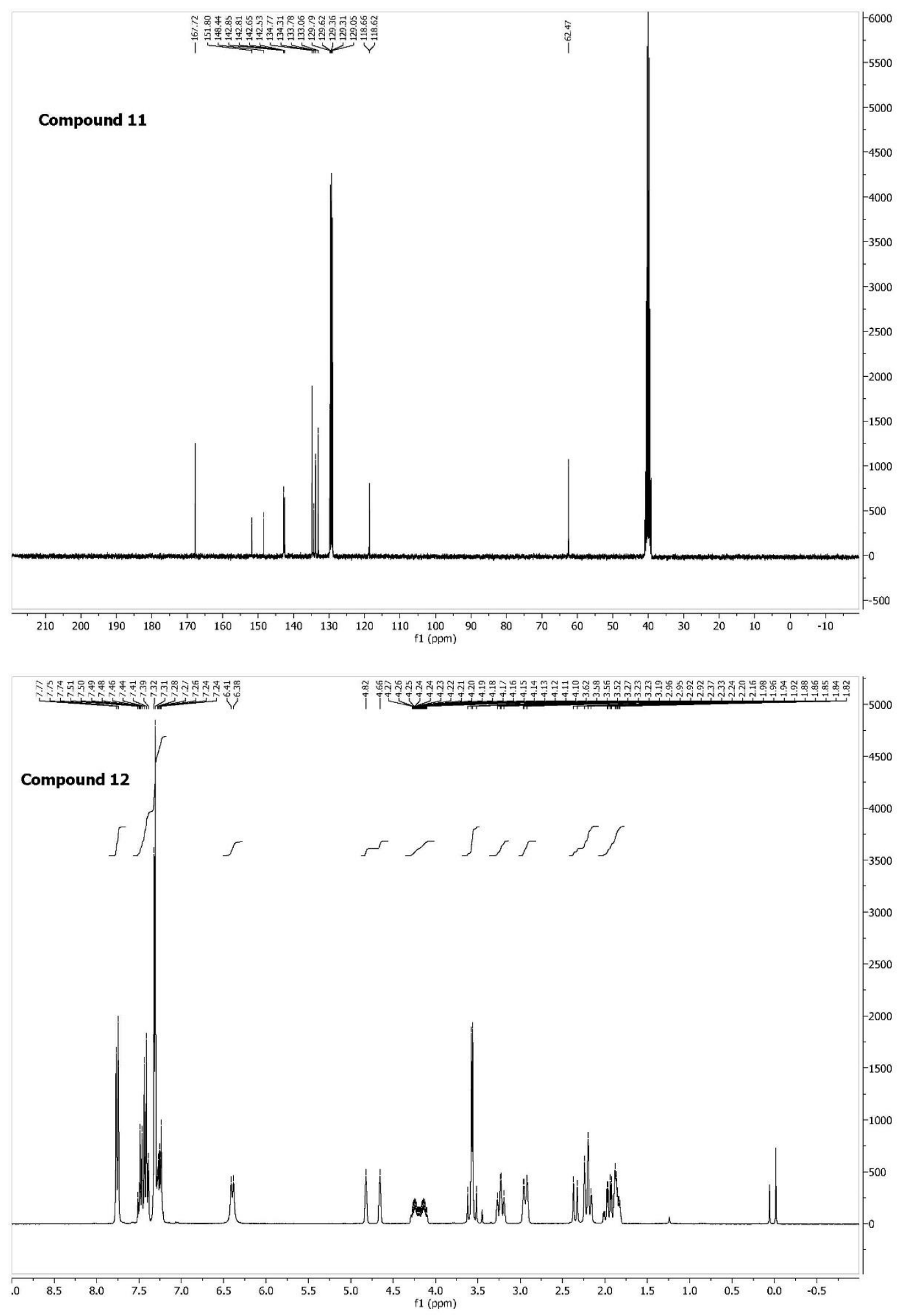



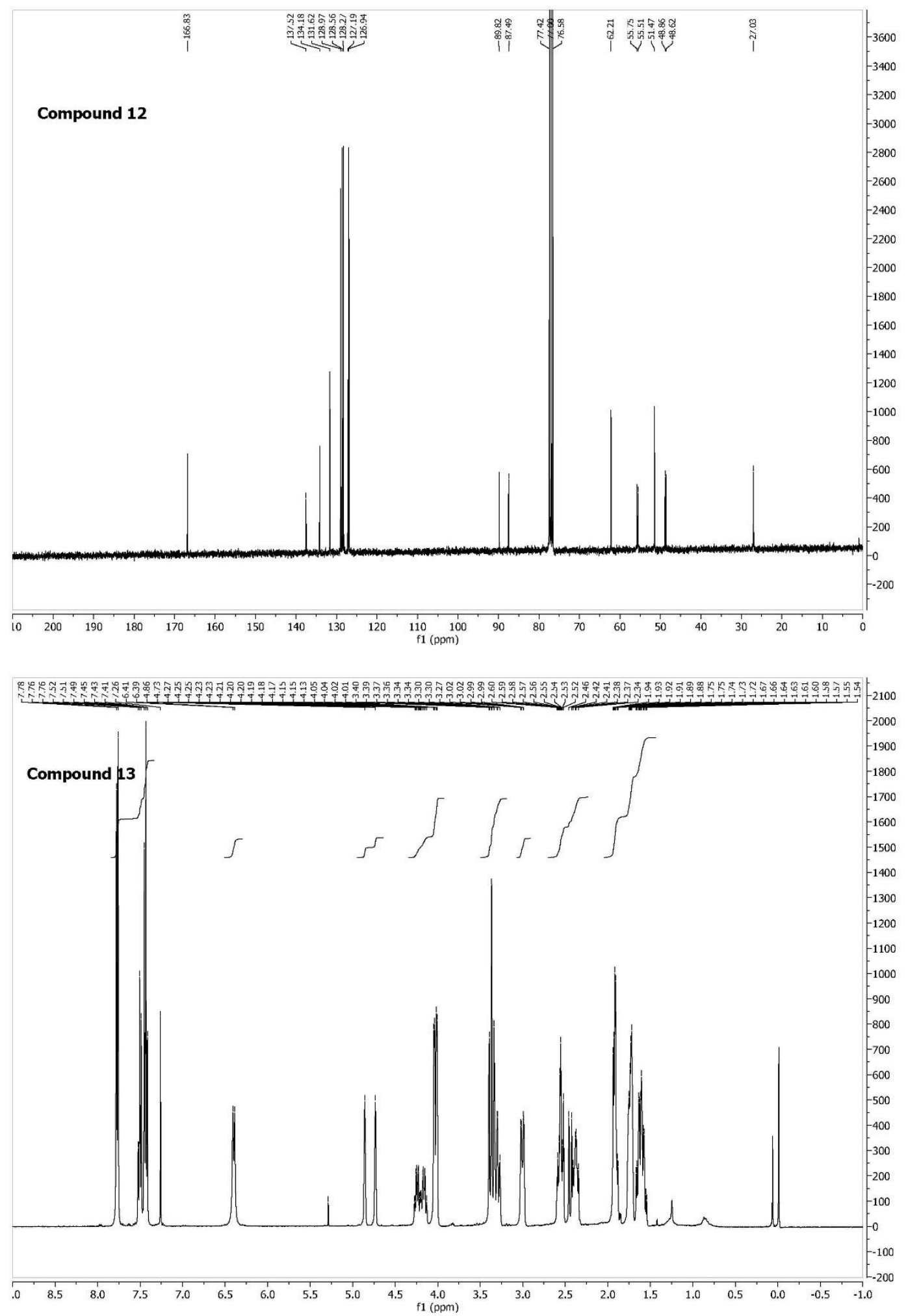

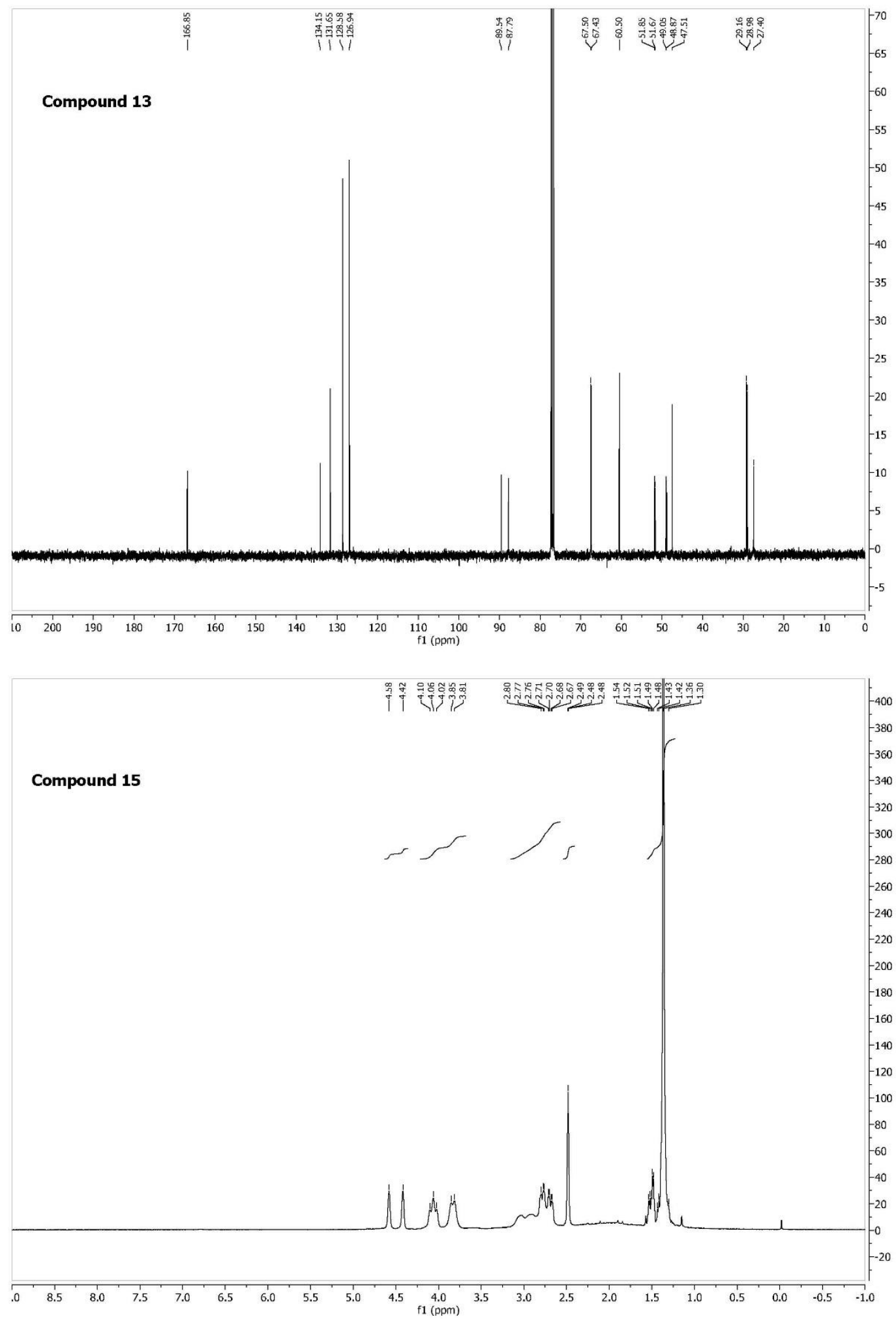


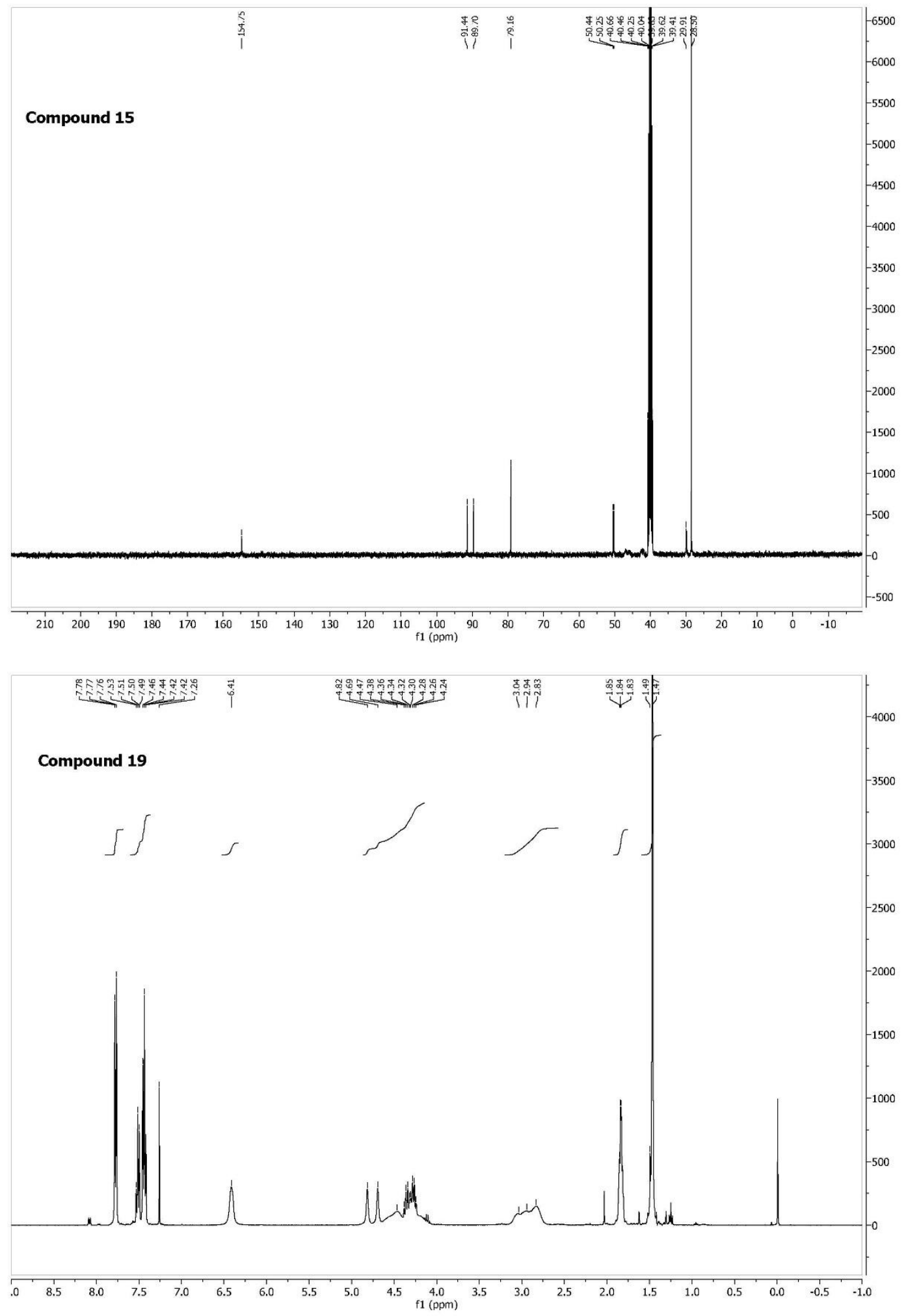



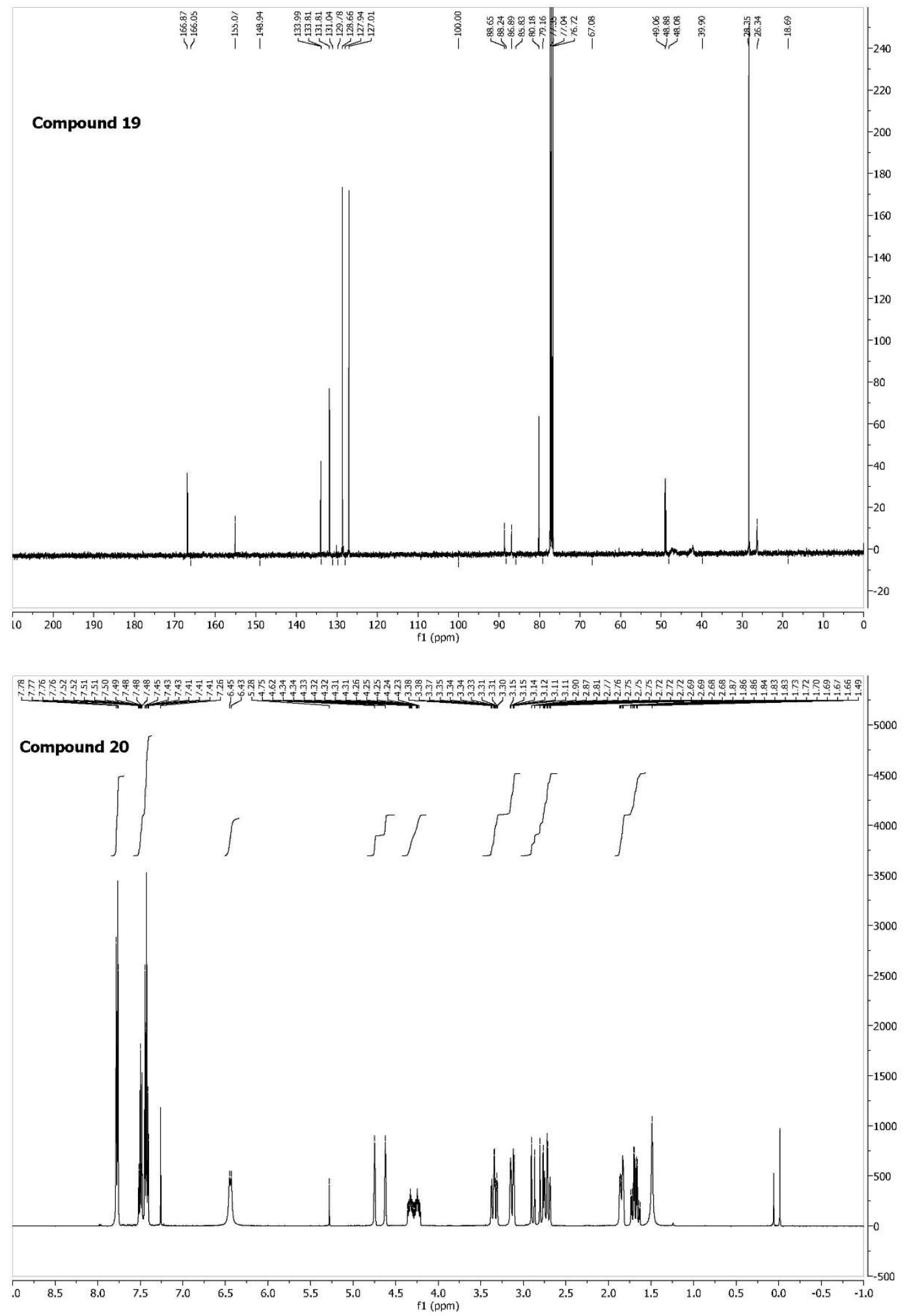

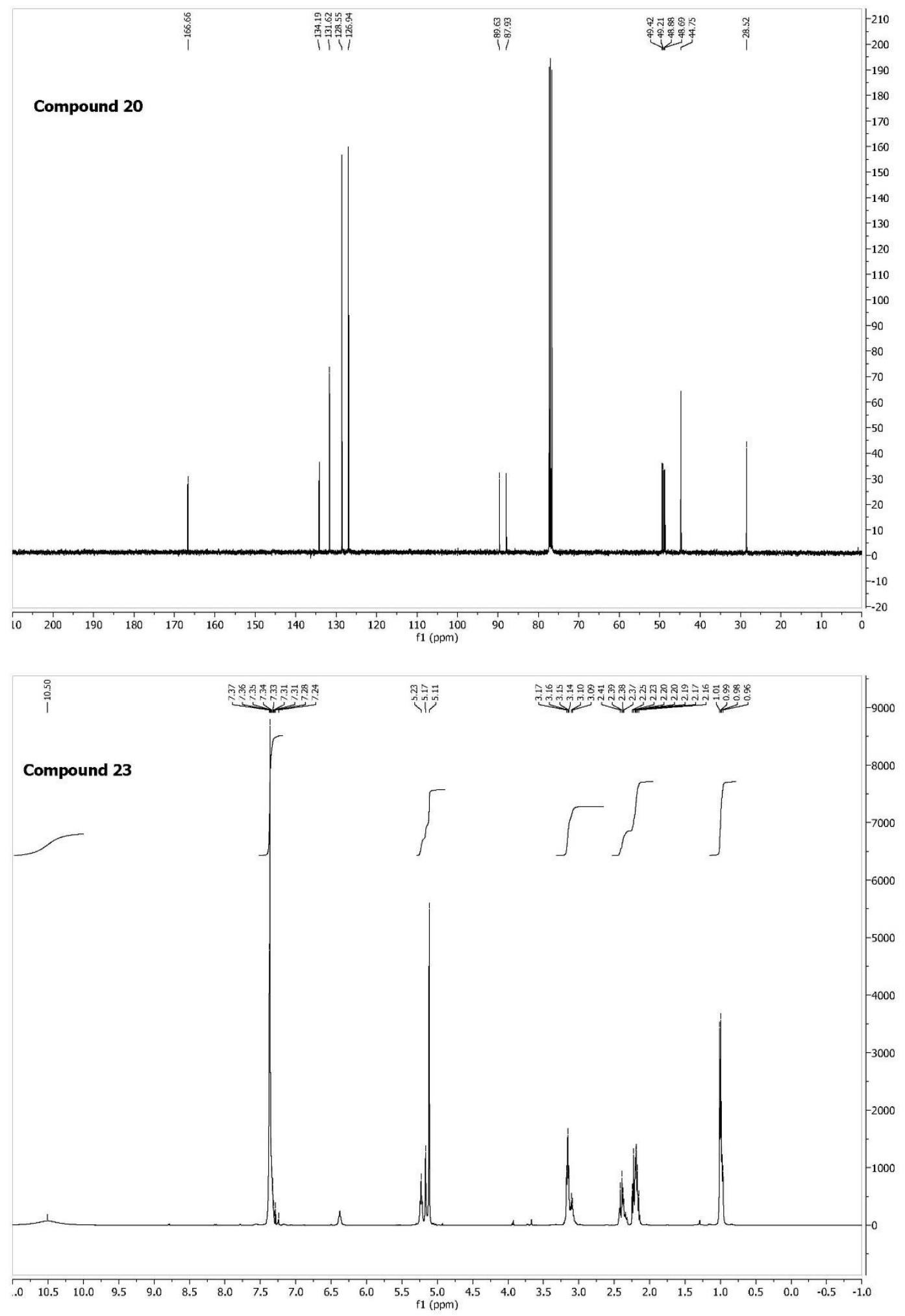

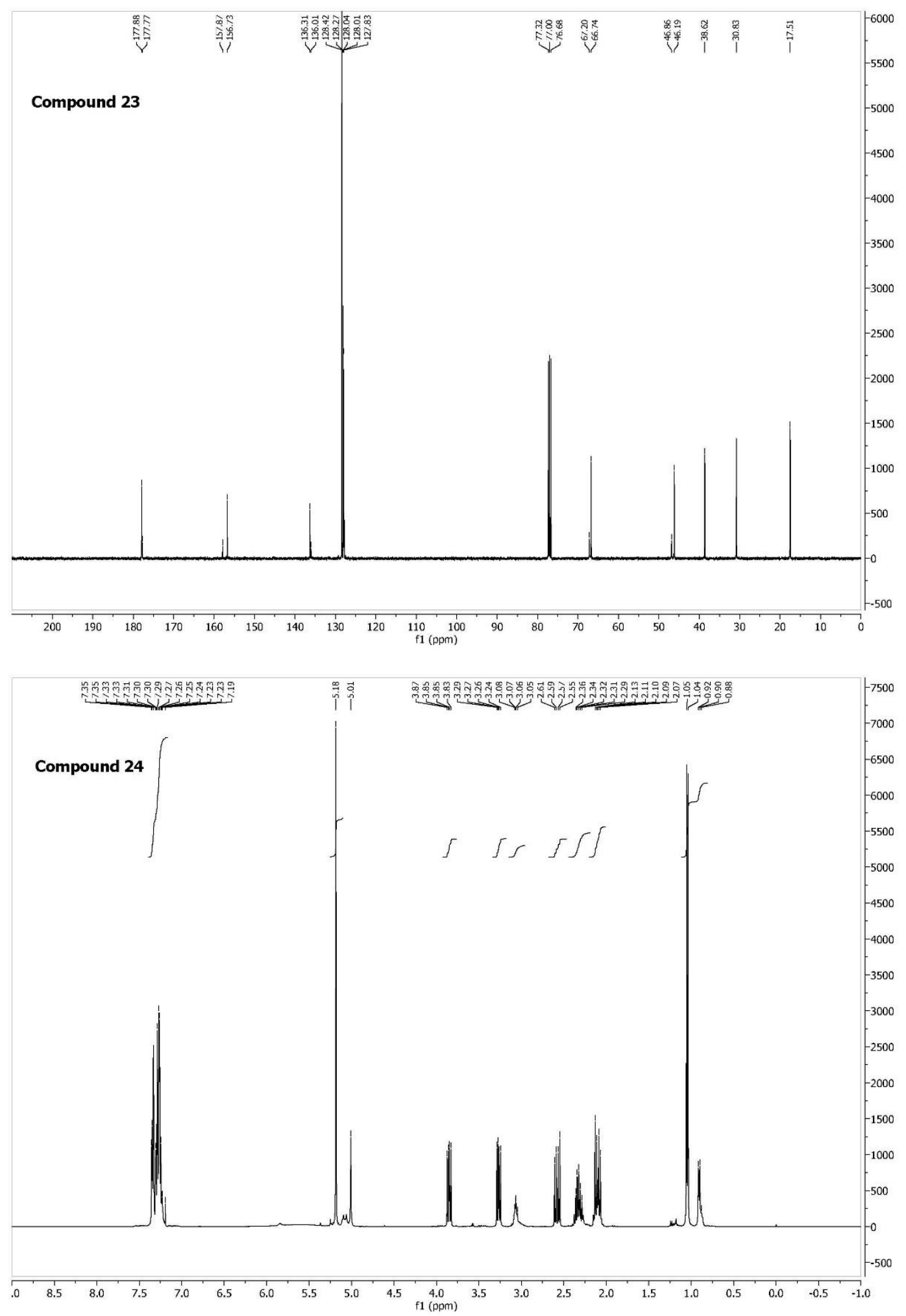

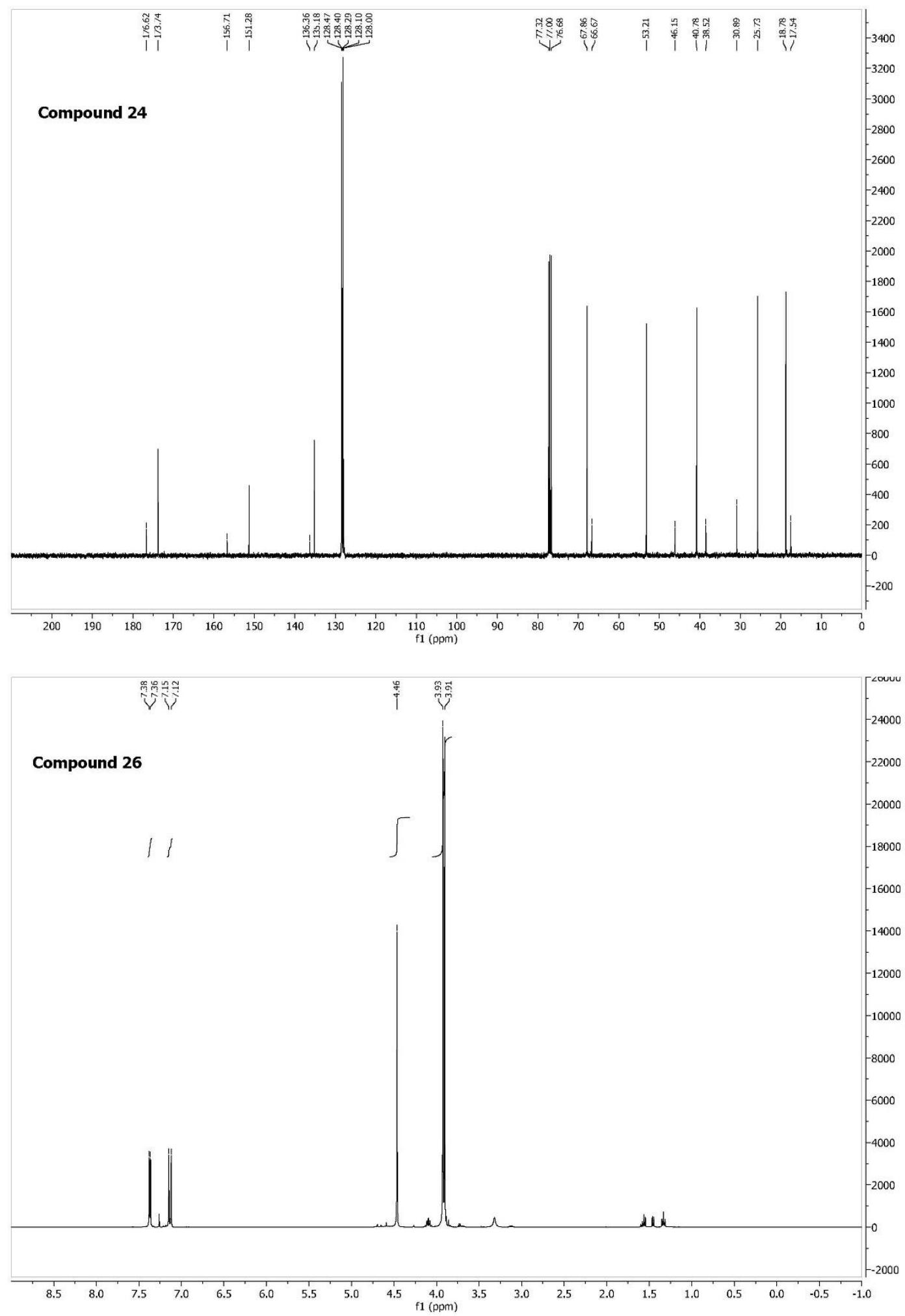

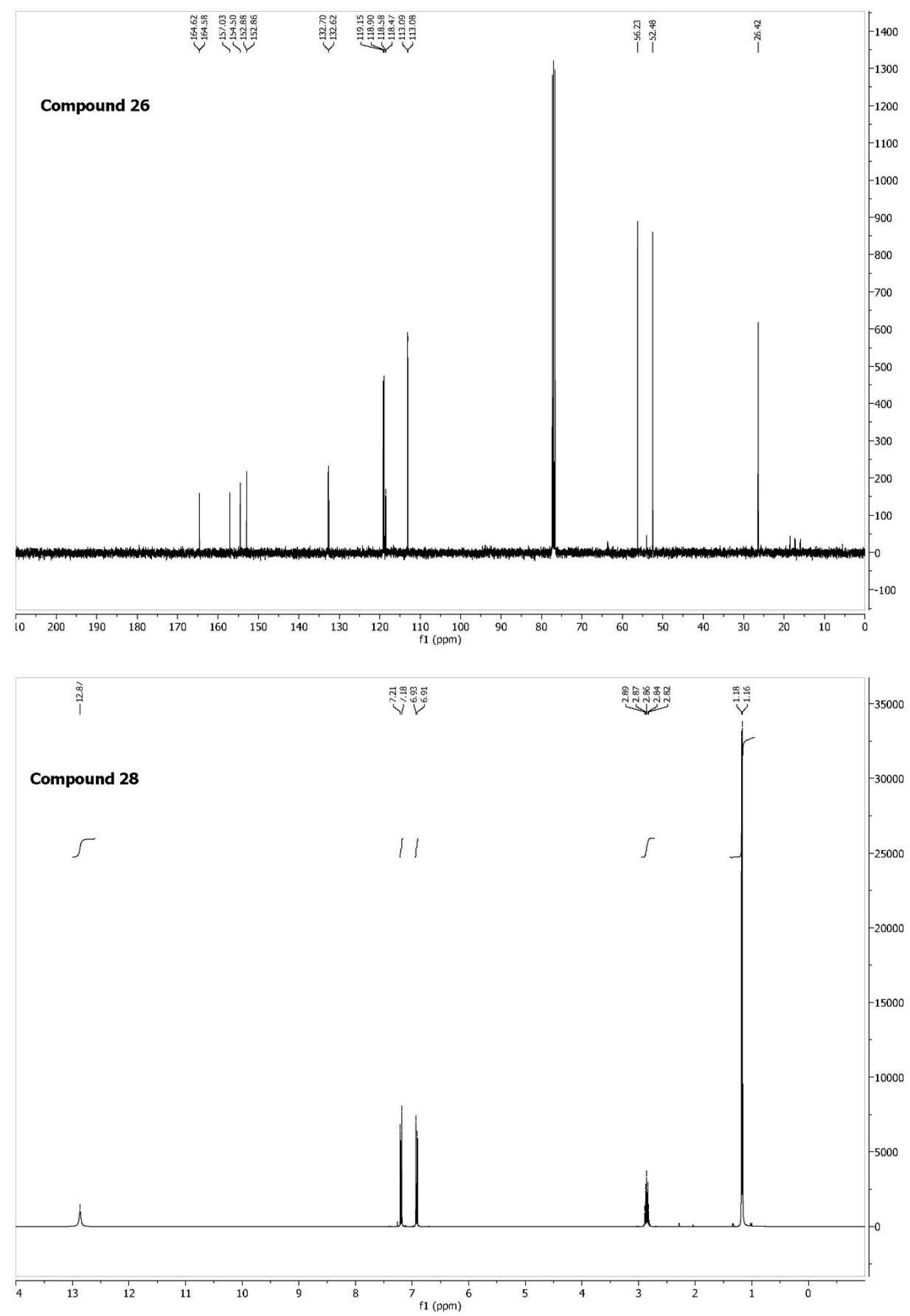

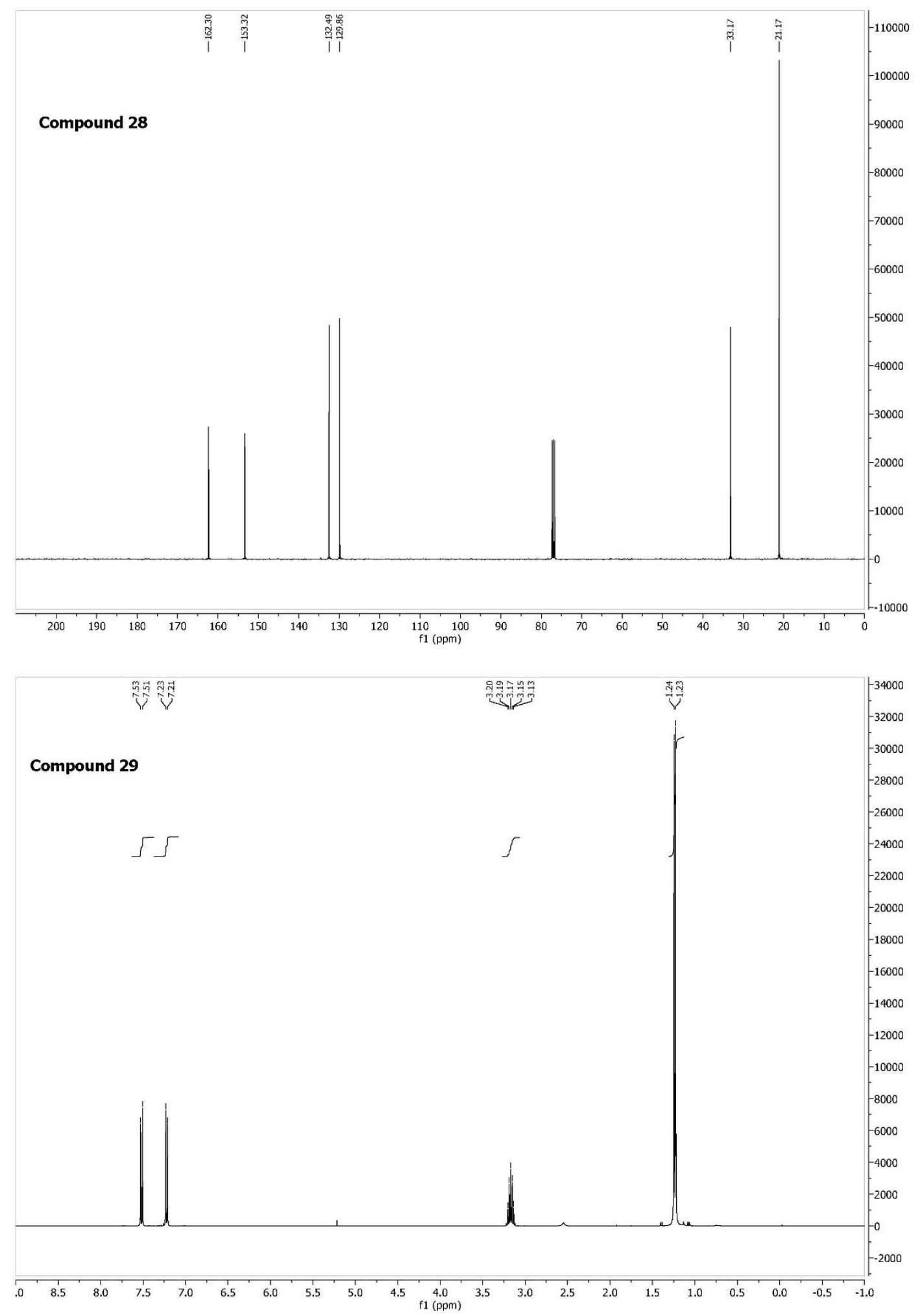

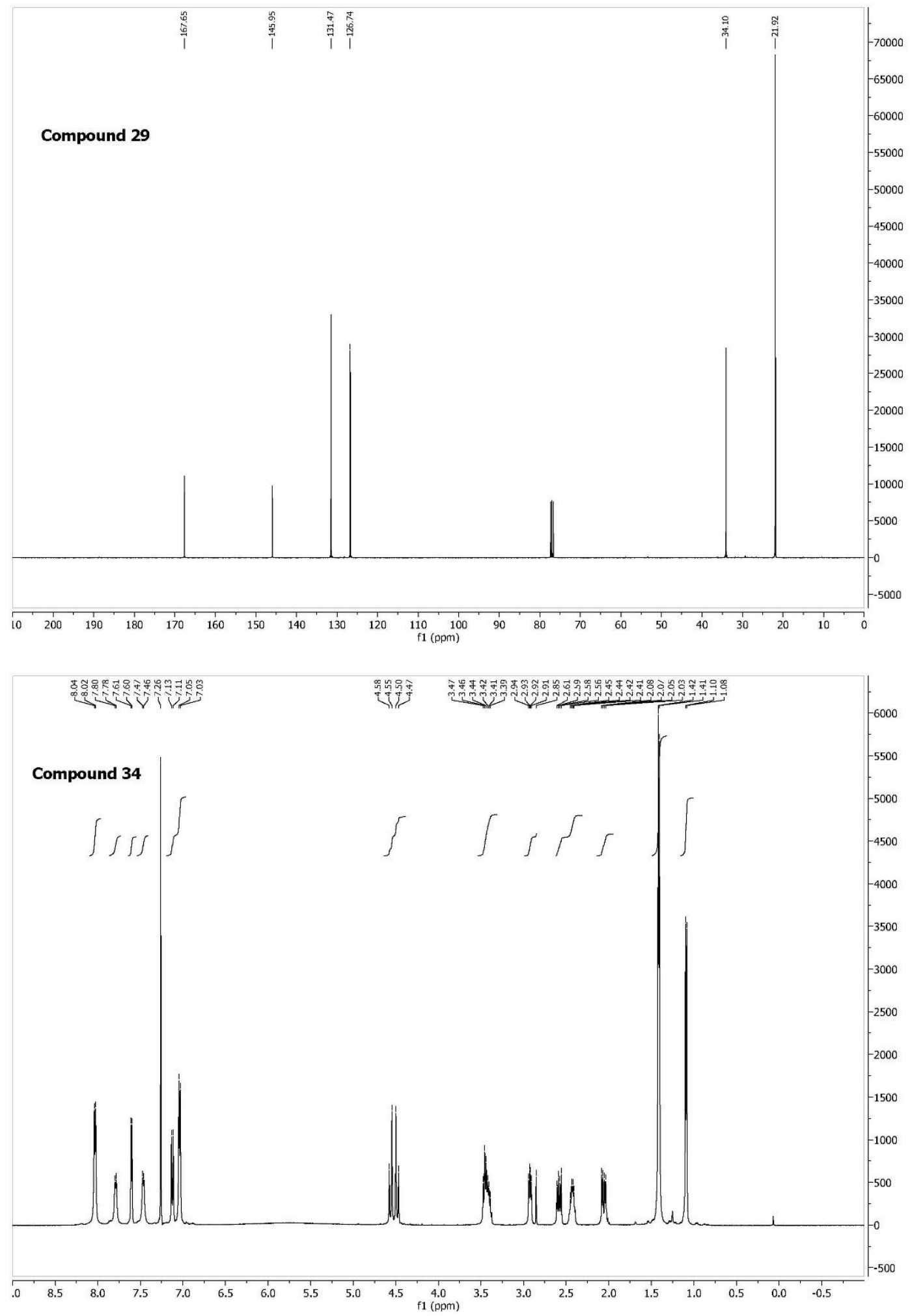


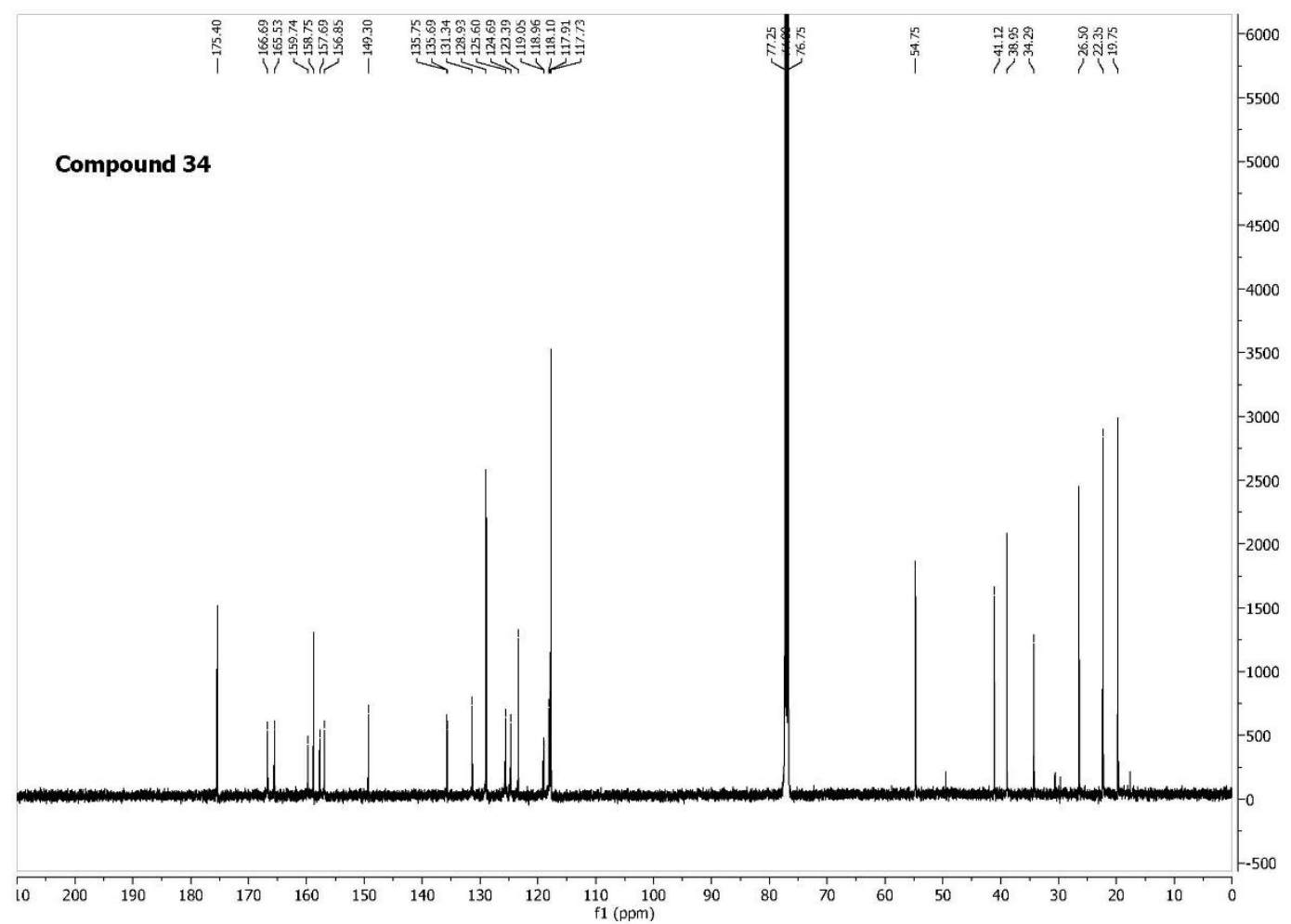




\section{Selected HPLC Chromatograms}

HPLC chromatogram for $\mathbf{1 5}$ racemate

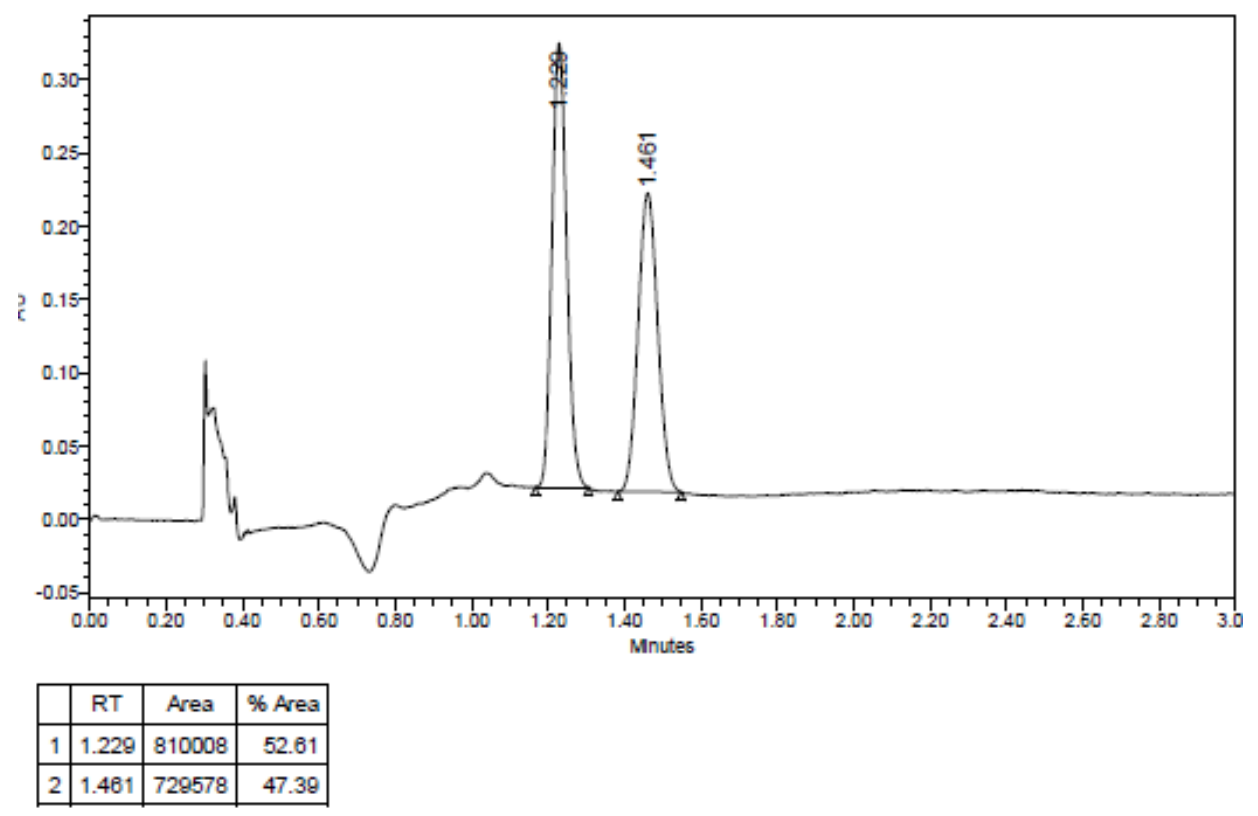

HPLC chromatogram for enantioenriched 15 (crude)

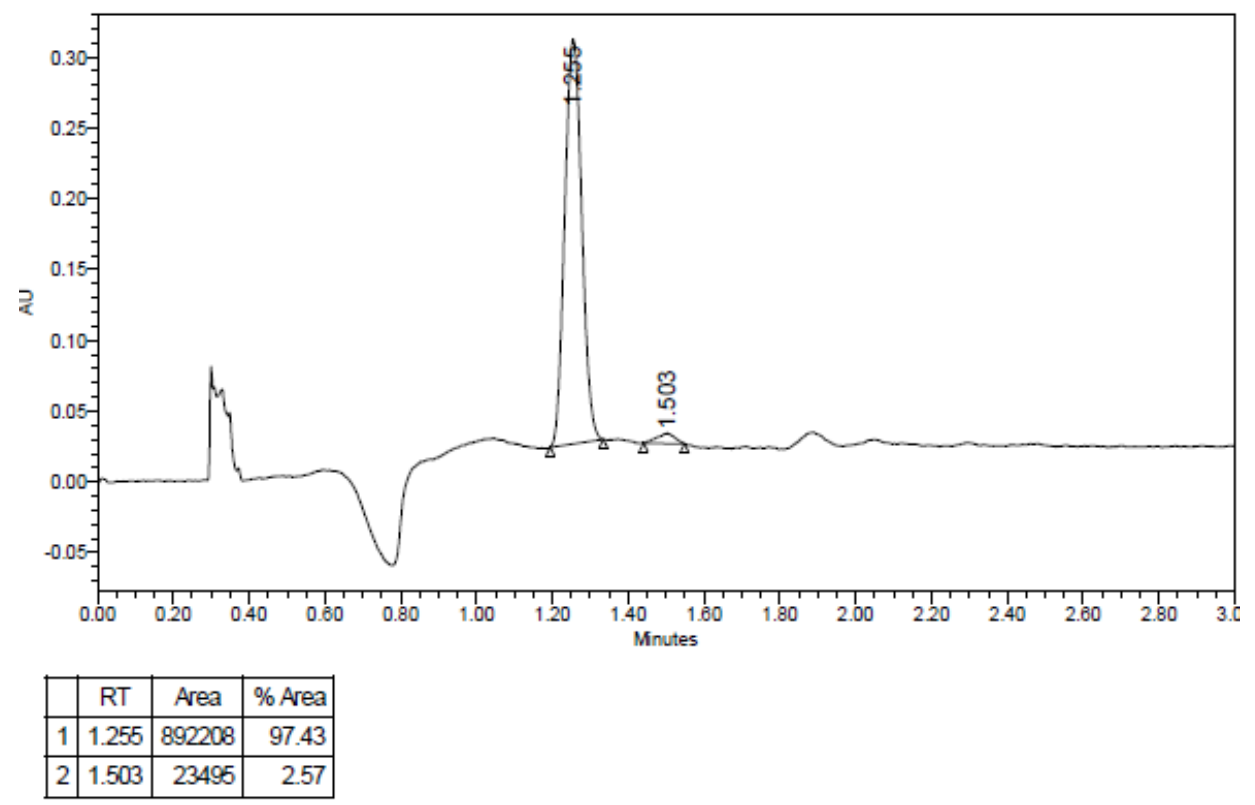


HPLC chromatogram for $\mathbf{1 2}$ racemate

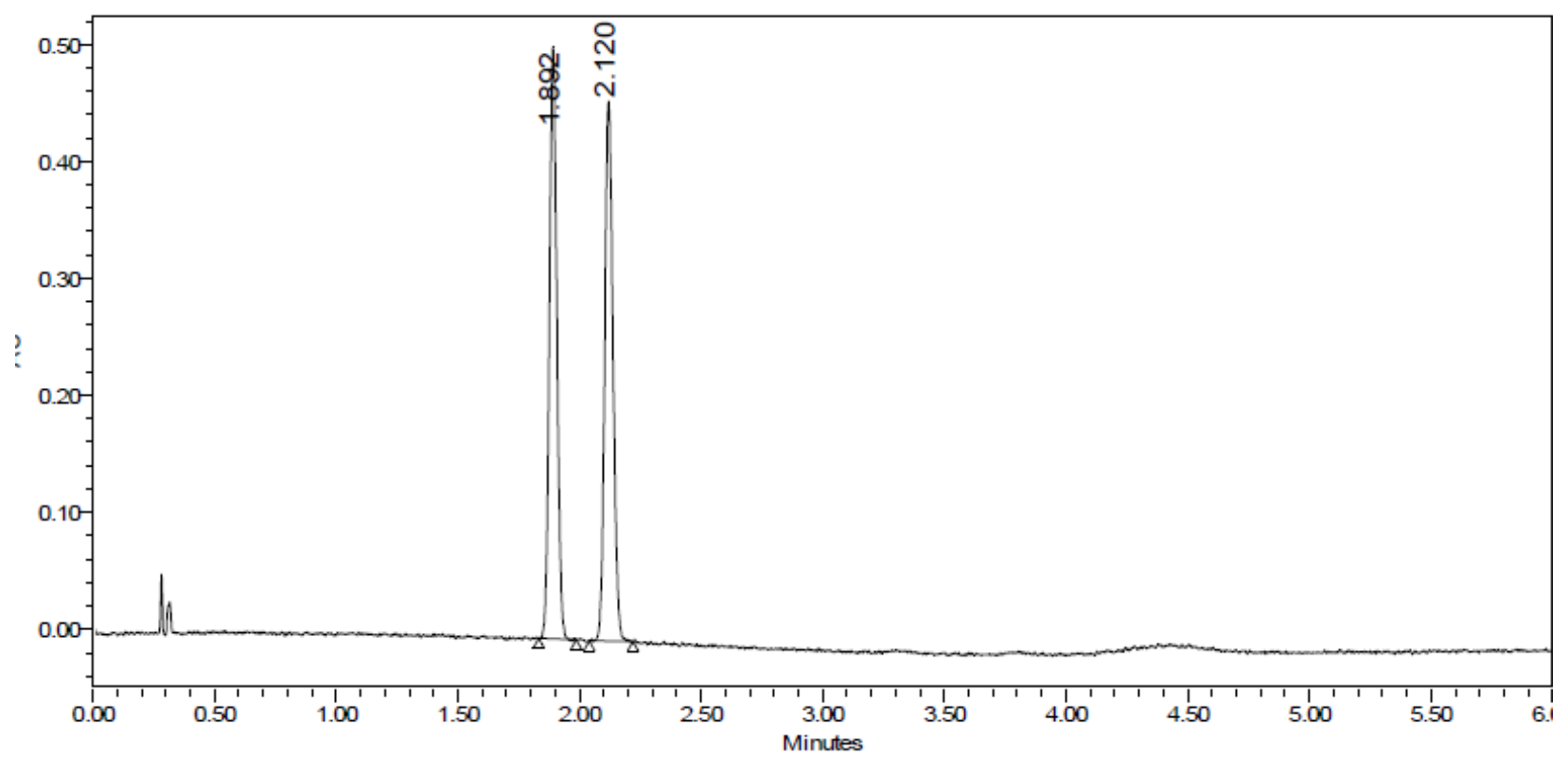

\begin{tabular}{|l|c|c|r|}
\hline & RT & Area & \% Area \\
\hline 1 & 1.892 & 1079640 & 49.80 \\
\hline 2 & 2.120 & 1088154 & 50.20 \\
\hline
\end{tabular}

HPLC chromatogram for enantioenriched 12 (purified)

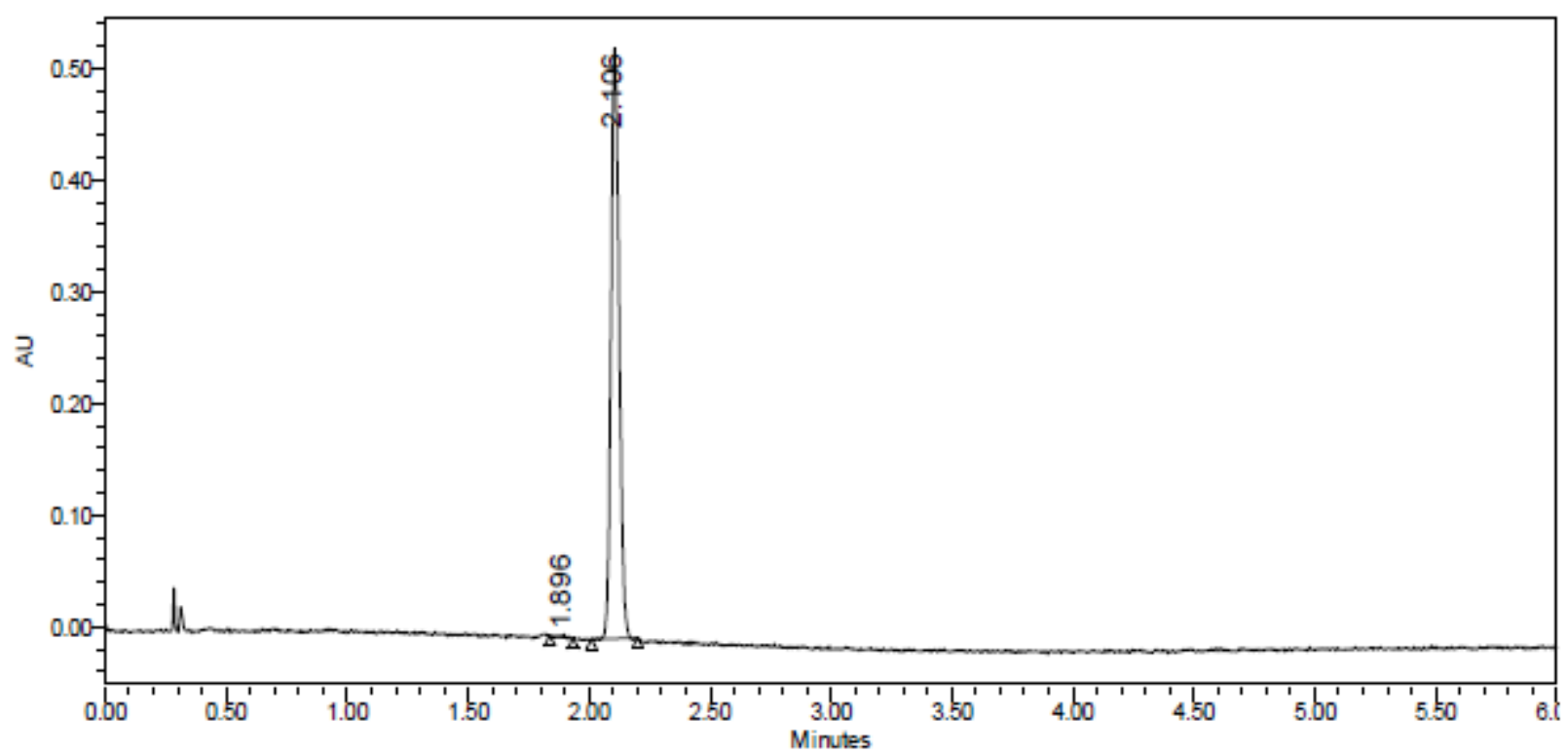

\begin{tabular}{|l|c|r|r|}
\hline & RT & \multicolumn{1}{|c|}{ Area } & $\%$ Area \\
\hline 1 & 1.896 & 4796 & 0.39 \\
\hline 2 & 2.106 & 1232191 & 99.61 \\
\hline
\end{tabular}

